\title{
Duplexing the Sponge: \\ Single-Pass Authenticated Encryption and Other Applications
}

\author{
Guido Bertoni ${ }^{1}$, Joan Daemen ${ }^{1}$, Michaël Peeters ${ }^{2}$, and Gilles Van Assche ${ }^{1}$ \\ 1 STMicroelectronics \\ 2 NXP Semiconductors
}

\begin{abstract}
This paper proposes a novel construction, called duplex, closely related to the sponge construction, that accepts message blocks to be hashed and - at no extra cost - provides digests on the input blocks received so far. It can be proven equivalent to a cascade of sponge functions and hence inherits its security against single-stage generic attacks. The main application proposed here is an authenticated encryption mode based on the duplex construction. This mode is efficient, namely, enciphering and authenticating together require only a single call to the underlying permutation per block, and is readily usable in, e.g., key wrapping. Furthermore, it is the first mode of this kind to be directly based on a permutation instead of a block cipher and to natively support intermediate tags. The duplex construction can be used to efficiently realize other modes, such as a reseedable pseudo-random bit sequence generators and a sponge variant that overwrites part of the state with the input block rather than to XOR it in.
\end{abstract}

Keywords: sponge functions, duplex construction, authenticated encryption, key wrapping, provable security, pseudo-random bit sequence generator, Keccak.

\section{Introduction}

While most symmetric-key modes of operations are based on a block cipher or a stream cipher, there exist modes using a fixed permutation as underlying primitive. Designing a cryptographically strong permutation suitable for such purposes is similar to designing a block cipher without a key schedule and this design approach was followed for several recent hash functions, see, e.g., 15.

The sponge construction is an example of such a mode. With its arbitrarily long input and output sizes, it allows building various primitives such as a stream cipher or a hash function [5]. In the former, the input is short (typically the key and a nonce) while the output is as long as the message to encrypt. In contrast, the latter takes a message of any length at input and produces a digest of small length.

Some applications can take advantage of both a long input and a long output size. For instance, authenticated encryption combines the encryption of a 
message and the generation of a message authentication code (MAC) on it. It could be implemented with one sponge function call to generate a key stream (long output) for the encryption and another call to generate the MAC (long input). However, in this case, encryption and authentication are separate processes without any synergy.

The duplex construction is a novel way to use a fixed permutation (or transformation) to allow the alternation of input and output blocks at the same rate as the sponge construction, like a full-duplex communication. In fact, the duplex construction can be seen as a particular way to use the sponge construction, hence it inherits its security properties. By using the duplex construction, authenticated encryption requires only one call to the underlying permutation (or transformation) per message block. In a nutshell, the input blocks of the duplex are used to input the key and the message blocks, while the intermediate output blocks are used as key stream and the last one as a MAC.

Authenticated encryption (AE) has been extensively studied in the last ten years. Block cipher modes clearly are a popular way to provide simultaneously both integrity and confidentiality. Many block cipher modes have been proposed and most of these come with a security proof against generic attacks - see [8] for references. Interestingly, there have also been attempts at designing dedicated hybrid primitives offering efficient simultaneous stream encryption and MAC computation, e.g., Helix and Phelix [1631]. However, these primitives were shown to be weak 22 24:32. Another example of hybrid primitive is the Grain-128 stream cipher to which optional built-in authentication was recently added 33.

Our proposed mode shares with these hybrid primitives that it offers efficient simultaneous stream encryption and MAC computation. It shares with the block cipher modes that it has provable security against generic attacks. However, it is the first such construction that (directly) relies on a permutation rather than a block cipher and that proves its security based on this type of primitive. An important efficiency parameter of an AE mode is the number of calls to the block cipher or to the permutation per block. While encryption or authentication alone requires one call per block, some $\mathrm{AE}$ modes only require one call per block for both functions. The duplex construction naturally provides a good basis for building such an efficient AE mode. Also, the AE mode we propose natively supports intermediate tags and the authenticated encryption of a sequence of messages.

Authenticated encryption can also be used to transport secret keys in a confidential way and to ensure their integrity. This task, called key wrapping, is very important in key management and can be implemented with our construction if each key has a unique identifier.

Finally, the duplex construction can be used for other modes as well, such as a reseedable pseudo-random bit sequence generator (PRG) or to prove the security of an "overwrite" mode where the input block overwrites part of the state instead of XORing it in. 
These modes can readily be used by the concrete sponge function KECCAK [10] and the members of a recent wave of lightweight hash functions that are in fact sponge functions: Quark [1, Photon [18] and Spongent [12. For these, and for the small-width instances of KECCAK, our security bound against generic attacks beyond the birthday bound published in [9] allows constructing solutions that are at the same time compact, efficient and potentially secure.

The remainder of this paper is organized as follows. First, we propose a model for authenticated encryption in Section 2. Then in Section 3. we review the sponge construction. The core concept of this paper, namely the duplex construction, is defined in Section 4. Its use for authenticated encryption is given in Section 5 and for other applications in Section 6. Finally, Section 7 discusses the use of a flexible and compact padding. For compactness reasons, the proofs are omitted in this version and can be found in [8].

\section{Modeling Authenticated Encryption}

We consider authenticated encryption as a process that takes as input a key $K$, a data header $A$ and a data body $B$ and that returns a cryptogram $C$ and a tag $T$. We denote this operation by the term wrapping and the operation of taking a data header $A$, a cryptogram $C$ and a tag $T$ and returning the data body $B$ if the $\operatorname{tag} T$ is correct by the term unwrapping.

The cryptogram is the data body enciphered under the key $K$ and the tag is a MAC computed under the same key $K$ over both header $A$ and body $B$. So here the header $A$ can play the role of associated data as described in [26]. We assume the wrapping and unwrapping operations as such to be deterministic. Hence two equal inputs $(A, B)=\left(A^{\prime}, B^{\prime}\right)$ will give rise to the same output $(C, T)$ under the same key $K$. If this is a problem, it can be tackled by expanding $A$ with a nonce.

Formally, for a given key length $k$ and tag length $t$, we consider a pair of algorithms $W$ and $U$, with

$$
\begin{gathered}
W: \mathbb{Z}_{2}^{k} \times\left(\mathbb{Z}_{2}^{*}\right)^{2} \rightarrow \mathbb{Z}_{2}^{*} \times \mathbb{Z}_{2}^{t}:(K, A, B) \rightarrow(C, T)=W(K, A, B), \text { and } \\
U: \mathbb{Z}_{2}^{k} \times\left(\mathbb{Z}_{2}^{*}\right)^{2} \times \mathbb{Z}_{2}^{t} \rightarrow \mathbb{Z}_{2}^{*} \cup\{\text { error }\}:(K, A, C, T) \rightarrow B \text { or error. }
\end{gathered}
$$

The algorithms are such that if $(C, T)=W(K, A, B)$ then $U(K, A, C, T)=B$. As we consider only the case of non-expanding encryption, we assume from now on that $|C|=|B|$.

\subsection{Intermediate Tags and Authenticated Encryption of a Sequence}

So far, we have only considered the case of the authentication and encryption of a single message, i.e., a header and body pair $(A, B)$. It can also be interesting to authenticate and encrypt a sequence of messages in such a way that the authenticity is guaranteed not only on each $(A, B)$ pair but also on the sequence received so far. Intermediate tags can also be useful in practice to be able to catch fraudulent transactions early. 
Let $(\overline{A, B})=\left(A^{(1)}, B^{(1)}, A^{(2)}, \ldots, A^{(n)}, B^{(n)}\right)$ be a sequence of header-body pairs. We extend the function of wrapping and unwrapping as providing encryption over the last body $B^{(n)}$ and authentication over the whole sequence $(\overline{A, B})$. Formally, $W$ and $U$ are defined as:

$$
\begin{gathered}
W: \mathbb{Z}_{2}^{k} \times\left(\mathbb{Z}_{2}^{*}\right)^{2+} \rightarrow \mathbb{Z}_{2}^{*} \times \mathbb{Z}_{2}^{t}:(K, \overline{A, B}) \rightarrow\left(C^{(\text {last })}, T^{(\text {last })}\right)=W(K, \overline{A, B}), \text { and } \\
U: \mathbb{Z}_{2}^{k} \times\left(\mathbb{Z}_{2}^{*}\right)^{2+} \times \mathbb{Z}_{2}^{t} \rightarrow \mathbb{Z}_{2}^{*} \cup\{\text { error }\}:\left(K, \overline{A, C}, T^{\text {(last) }) \rightarrow B^{(\text {last })} \text { or error } .}\right.
\end{gathered}
$$

Here, $\left(\mathbb{Z}_{2}^{*}\right)^{2+}$ means any sequence of binary strings, with an even number of such strings and at least two. To wrap a sequence of header-body pairs, the sender calls $W\left(K, A^{(1)}, B^{(1)}\right)$ with the first header-body pair to get $\left(C^{(1)}, T^{(1)}\right)$, then $W\left(K, A^{(1)}, B^{(1)}, A^{(2)}, B^{(2)}\right)$ with the second one to get $\left(C^{(2)}, T^{(2)}\right)$, and so on. To unwrap, the receiver first calls $U\left(K, A^{(1)}, C^{(1)}, T^{(1)}\right)$ to retrieve the first body $B^{(1)}$, then $U\left(K, A^{(1)}, C^{(1)}, A^{(2)}, C^{(2)}, T^{(2)}\right)$ to retrieve the second body, and so on. As we consider only the case of non-expanding encryption, we assume that $\left|C^{(i)}\right|=\left|B^{(i)}\right|$ for all $i$.

\subsection{Security Requirements}

We consider two security notions from 28, and works cited therein, called privacy and authenticity. Together, these notions are central to the security of authenticated encryption [2].

Privacy is defined in Eq. (11) below. Informally, it means that the output of the wrapping function looks like uniformly chosen random bits to an observer who does not know the key.

$$
\operatorname{Adv}^{\operatorname{priv}}(\mathcal{A})=\left|\operatorname{Pr}\left[K \stackrel{\$}{\leftarrow} \mathbb{Z}_{2}^{k}: \mathcal{A}[W(K, \cdot, \cdot)]=1\right]-\operatorname{Pr}[\mathcal{A}[R(\cdot, \cdot)]=1]\right|,
$$

with $R(\overline{A, B})=\lfloor\mathcal{R O}(\overline{A, B})\rfloor_{\left|B^{(n)}\right|+t}$ where $B^{(n)}$ is the last body in $\overline{A, B},|x|$ is the bitlength of string $x,\lrcorner_{\ell}$ indicates truncation to $\ell$ bits and $K \stackrel{\$}{\leftarrow} \mathbb{Z}_{2}^{k}$ means that $K$ is chosen randomly and uniformly among the set $\mathbb{Z}_{2}^{k}$. In this definition, we use a random oracle $\mathcal{R O}$ as defined in [3], but allowing sequences of one or more binary strings as input (instead of a single binary string). Here, a random oracle is a map from $\left(\mathbb{Z}_{2}^{*}\right)^{+}$to $\mathbb{Z}_{2}^{\infty}$, chosen by selecting each bit of $\mathcal{R O}(x)$ uniformly and independently, for every input. The original definition can still be used by defining an injective mapping from $\left(\mathbb{Z}_{2}^{*}\right)^{+}$to $\mathbb{Z}_{2}^{*}$.

For privacy, we consider only adversaries who respect the nonce requirement. For a single header-body pair, it means that, for any two queries $(A, B)$ and $\left(A^{\prime}, B^{\prime}\right)$, we have $A=A^{\prime} \Rightarrow B=B^{\prime}$. In general, the nonce requirement specifies that for any two queries $(\overline{A, B})$ and $\left(\overline{A^{\prime}, B^{\prime}}\right)$ of equal length $n$, we have

$$
\operatorname{pre}(\overline{A, B})=\operatorname{pre}\left(\overline{A^{\prime}, B^{\prime}}\right) \Rightarrow B^{(n)}=B^{\prime(n)},
$$


with pre $(\overline{A, B})=\left(A^{(1)}, B^{(1)}, A^{(2)}, \ldots, B^{(n-1)}, A^{(n)}\right)$ the sequence with the last body omitted. As for a stream cipher, not respecting the nonce requirement means that the adversary can learn the bitwise difference between two plaintext bodies.

Authenticity is defined in Eq. (22) below. Informally, it quantifies the probability of the adversary successfully generating a forged ciphertext-tag pair.

$$
\operatorname{Adv}^{\text {auth }}(\mathcal{A})=\operatorname{Pr}\left[K \stackrel{\$}{\leftarrow} \mathbb{Z}_{2}^{k}: \mathcal{A}[W(K, \cdot, \cdot)] \text { outputs a forgery }\right] .
$$

Here a forgery is a sequence $(\overline{A, C}, T)$ such that $U(K, \overline{A, C}, T) \neq$ error and that the adversary made no query to $W$ with input $(\overline{A, B})$ returning $\left(C^{(n)}, T\right)$, with $C^{(n)}$ the last ciphertext body of $\overline{A, C}$. Note that authenticity does not need the nonce requirement.

\subsection{An Ideal System}

We can define an ideal system using a pair of independent random oracles $\left(\mathcal{R O}_{\mathrm{C}}, \mathcal{R O}_{\mathrm{T}}\right)$. For a single header-body pair, encryption and tag computation are implemented as follows. The ciphertext $C$ is produced by XORing $B$ with a key stream. This key stream is the output of $\mathcal{R} \mathcal{O}_{\mathrm{C}}(K, A)$. If $(K, A)$ is a nonce, key streams for different data inputs are the result of calls to $\mathcal{R} \mathcal{O}_{\mathrm{C}}$ with different inputs and hence one key stream gives no information on another. The tag $T$ is the output of $\mathcal{R O}_{\mathrm{T}}(K, A, B)$. Tags computed over different header-body pairs will be the result of calls to $\mathcal{R O}_{\mathrm{T}}$ with different inputs. Key stream sequences give no information on tags and vice versa as they are obtained by calls to different random oracles.

Let us define the ideal system in the general case, which we call ROwRAP. Wrapping is defined as $W(K, \overline{A, B})=\left(C^{(n)}, T^{(n)}\right)$, if $\overline{A, B}$ contains $n$ headerbody pairs, with

$$
\begin{aligned}
C^{(n)} & =\left\lfloor\mathcal{R} \mathcal{O}_{\mathrm{C}}(K, \operatorname{pre}(\overline{A, B}))\right\rfloor_{\left|B^{(n)}\right|} \oplus B^{(n)}, \\
T^{(n)} & =\left\lfloor\mathcal{R} \mathcal{O}_{\mathrm{T}}(K, \overline{A, B})\right\rfloor_{t} .
\end{aligned}
$$

The unwrapping algorithm $U$ first checks that $T^{(n)}=\left\lfloor\mathcal{R} \mathcal{O}_{\mathrm{T}}(K, \overline{A, B})\right\rfloor_{t}$ and if so decrypts each body $B^{(i)}=\left\lfloor\mathcal{R} \mathcal{O}_{\mathrm{C}}\left(K, A^{(1)}, B^{(1)}, A^{(2)}, \ldots, A^{(i)}\right)\right\rfloor_{\left|C^{(i)}\right|} \oplus C^{(i)}$ from the first one to the last one and finally returns the last one $B^{(n)}=$ $\left\lfloor\mathcal{R} \mathcal{O}_{\mathrm{C}}(K, \operatorname{pre}(\overline{A, B}))\right\rfloor_{\left|C^{(n)}\right|} \oplus C^{(n)}$.

The security of ROWRAP is captured by Lemmas 1 and 2 .

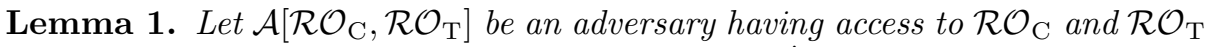
and respecting the nonce requirement. Then, $\operatorname{Adv}_{\mathrm{ROWRAP}}^{\text {priv }}(\mathcal{A}) \leq q 2^{-k}$ if the adversary makes no more than q queries to $\mathcal{R O}_{\mathrm{C}}$ or $\mathcal{R} \mathcal{O}_{\mathrm{T}}$.

Lemma 2. Let $\mathcal{A}\left[\mathcal{R O}_{\mathrm{C}}, \mathcal{R \mathcal { O } _ { \mathrm { T } }}\right]$ be an adversary having access to $\mathcal{R} \mathcal{O}_{\mathrm{C}}$ and $\mathcal{R \mathcal { O } _ { \mathrm { T } }}$. Then, ROWRAP satisfies $\operatorname{Adv}_{\text {ROWRAP }}(\mathcal{A}) \leq q 2^{-k}+2^{-t}$ if the adversary makes no more than q queries to $\mathcal{R O}_{\mathrm{C}}$ or $\mathcal{R O}_{\mathrm{T}}$. 


\section{The Sponge Construction}

The sponge construction [5] builds a function SPONGE[ $f, \operatorname{pad}, r]$ with variablelength input and arbitrary output length using a fixed-length permutation (or transformation) $f$, a padding rule "pad" and a parameter bitrate $r$.

For the padding rule we use the following notation: the padding of a message $M$ to a sequence of $x$-bit blocks is denoted by $M \| \operatorname{pad}[x](|M|)$, where $|M|$ is the length of $M$. This notation highlights that we only consider padding rules that append a bitstring that is fully determined by the length of $M$ and the block length $x$. We may omit $[x],|M|$ or both if their value is clear from the context.

Definition 1. A padding rule is sponge-compliant if it never results in the empty string and if it satisfies following criterion:

$$
\forall n \geq 0, \forall M, M^{\prime} \in \mathbb{Z}_{2}^{*}: M \neq M^{\prime} \Rightarrow M\left\|\operatorname{pad}[r](|M|) \neq M^{\prime}\right\| \operatorname{pad}[r]\left(\left|M^{\prime}\right|\right) \| 0^{n r}
$$

For the sponge construction to be secure (see Section 3.2), the padding rule pad must be sponge-compliant. As a sufficient condition, a padding rule that is reversible, non-empty and such that the last block must be non-zero, is spongecompliant [5].

\subsection{Definition}

The permutation $f$ operates on a fixed number of bits, the width $b$. The sponge construction has a state of $b$ bits. First, all the bits of the state are initialized to zero. The input message is padded with the function $\operatorname{pad}[r]$ and cut into $r$-bits blocks. Then it proceeds in two phases: the absorbing phase followed by the squeezing phase. In the absorbing phase, the $r$-bit input message blocks are XORed into the first $r$ bits of the state, interleaved with applications of the function $f$. When all message blocks are processed, the sponge construction switches to the squeezing phase. In the squeezing phase, the first $r$ bits of the state are returned as output blocks, interleaved with applications of the function $f$. The number of iterations is determined by the requested number of bits. Finally the output is truncated to the requested length. Algorithm 1 provides a formal definition.

The value $c=b-r$ is called the capacity. The last $c$ bits of the state are never directly affected by the input blocks and are never output during the squeezing phase. The capacity $c$ actually determines the attainable security level of the construction [6]9].

\subsection{Security}

Cryptographic functions are often designed in two steps. In the first step, one chooses a construction that uses a cryptographic primitive with fixed input and output size (e.g., a compression function or a permutation) and builds a function 


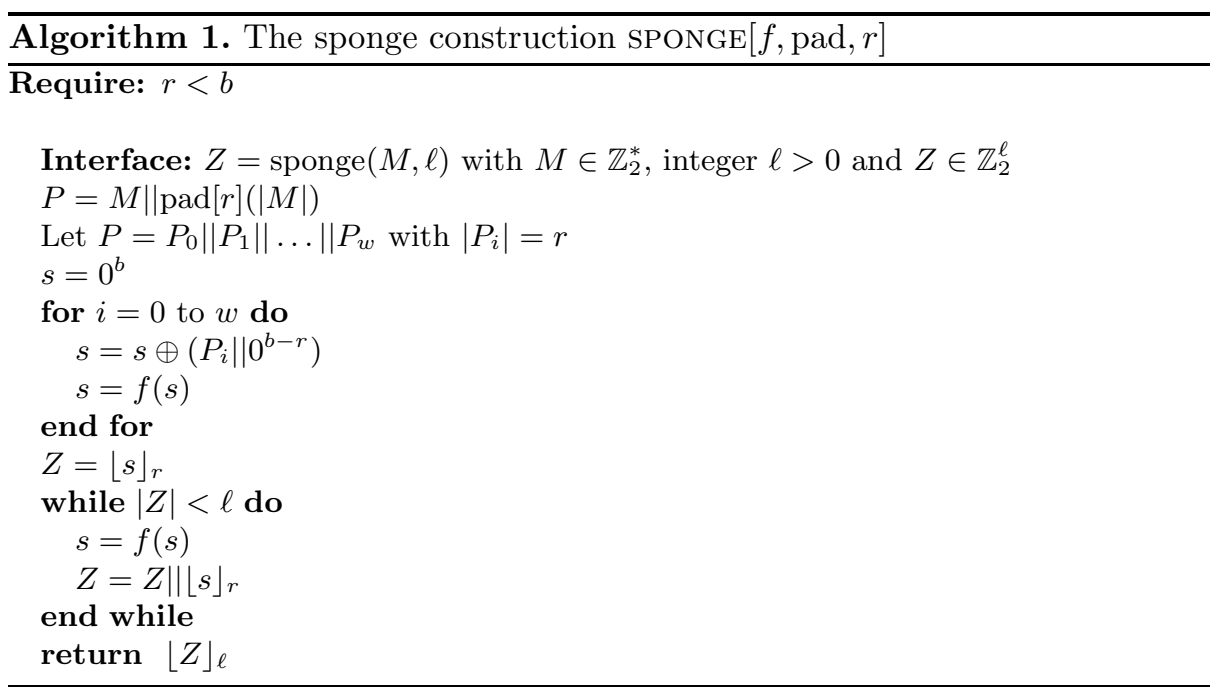

that can take inputs and or generate outputs of arbitrary size. If the security of this construction can be proven, for instance as in this case using the indifferentiability framework, it reduces the scope of cryptanalysis to that of the underlying primitive and guarantees the absence of single-stage generic attacks (e.g., preimage, second preimage and collision attacks) 21. However, generic security in the multi-stage setting using the indifferentiability framework is currently an open problem [25].

It is shown in [6] that the success probability of any single-stage generic attack for differentiating the sponge construction calling a random permutation or transformation from a random oracle is upper bounded by $2^{-(c+1)} N^{2}$. Here $N$ is the number of calls to the underlying permutation or its inverse. This implies that any single-stage generic attack on a sponge function has success probability of at most $2^{-(c+1)} N^{2}$ plus the success probability of this attack on a random oracle.

In [9], we address the security of the sponge construction when the message is prefixed with a key, as it will be done in the mode of Section 5. In this specific case, the security proof goes beyond the $2^{c / 2}$ complexity if the number of input or output blocks for which the key is used (data complexity) is upper bounded by $M<2^{c / 2-1}$. In that case, distinguishing the keyed sponge from a random oracle has time complexity of at least $2^{c-1} / M>2^{c / 2}$. Hence, for keyed modes, one can reduce the capacity $c$ for the same targeted security level.

\subsection{Implementing Authenticated Encryption}

The simplest way to build an actual system that behaves as ROWRAP would be to replace the random oracles $\mathcal{R \mathcal { O } _ { \mathrm { C } }}$ and $\mathcal{R \mathcal { O } _ { \mathrm { T } }}$ by a sponge function with domain separation. However, such a solution requires two sponge function executions: one for the generation of the key stream and one for the generation 
of the tag, while we aim for a single-pass solution. To achieve this, we define a variant where the key stream blocks and tag are the responses of a sponge function to input sequences that are each other's prefix. This introduces a new construction that is closely related to the sponge construction: the duplex construction. Subsequently, we build an authenticated encryption mode on top of that.

\section{The Duplex Construction}

Like the sponge construction, the duplex construction DUPLEx [f, pad, $r]$ uses a fixed-length transformation (or permutation) $f$, a padding rule "pad" and a parameter bitrate $r$. Unlike a sponge function that is stateless in between calls, the duplex construction accepts calls that take an input string and return an output string depending on all inputs received so far. We call an instance of the duplex construction a duplex object, which we denote $D$ in our descriptions. We prefix the calls made to a specific duplex object $D$ by its name $D$ and a dot.

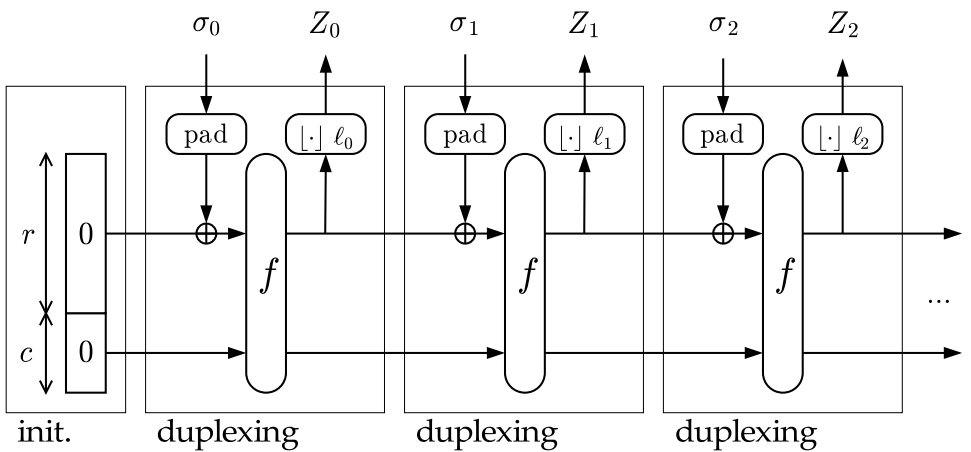

Fig. 1. The duplex construction

The duplex construction works as follows. A duplex object $D$ has a state of $b$ bits. Upon initialization all the bits of the state are set to zero. From then on one can send to it D.duplexing $(\sigma, \ell)$ calls, with $\sigma$ an input string and $\ell$ the requested number of bits.

The maximum number of bits $\ell$ one can request is $r$ and the input string $\sigma$ shall be short enough such that after padding it results in a single $r$-bit block. We call the maximum length of $\sigma$ the maximum duplex rate and denote it by $\rho_{\max }(\operatorname{pad}, r)$. Formally:

$$
\rho_{\max }(\operatorname{pad}, r)=\min \{x: x+|\operatorname{pad}[r](x)|>r\}-1 .
$$

Upon receipt of a $D$.duplexing $(\sigma, \ell)$ call, the duplex object pads the input string $\sigma$ and XORs it into the first $r$ bits of the state. Then it applies $f$ to the state 


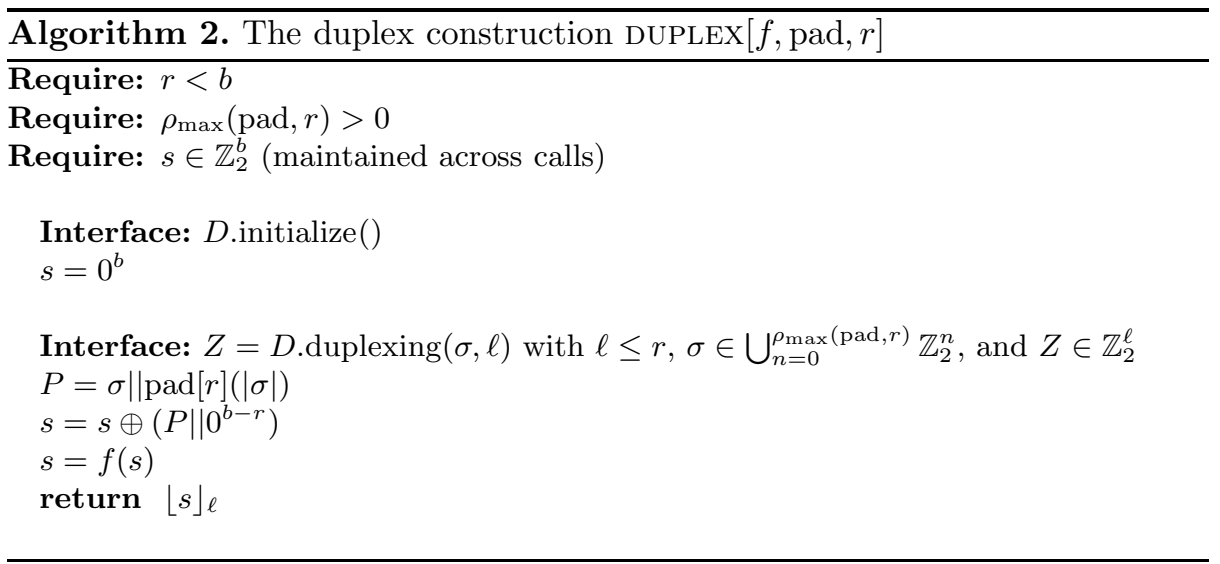

and returns the first $\ell$ bits of the state at the output. We call a blank call a call with $\sigma$ the empty string, and a mute call a call without output, $\ell=0$. The duplex construction is illustrated in Figure 1 and Algorithm 2 provides a formal definition.

The following lemma links the security of the duplex construction to that of the sponge construction with the same parameters, i.e., DUPLEX $[f$, pad, $r]$ and SPONGE $[f$, pad, $r]$. Generating the output of a D.duplexing() call using a sponge function is illustrated in Figure 2 .

Lemma 3. [Duplexing-sponge lemma] If we denote the input to the $i$-th call to a duplex object by $\left(\sigma_{i}, \ell_{i}\right)$ and the corresponding output by $Z_{i}$ we have:

$$
Z_{i}=D \cdot \operatorname{duplexing}\left(\sigma_{i}, \ell_{i}\right)=\operatorname{sponge}\left(\sigma_{0}\left\|\operatorname{pad}_{0}\right\| \sigma_{1}\left\|\operatorname{pad}_{1}\right\| \ldots \| \sigma_{i}, \ell_{i}\right)
$$

with $\operatorname{pad}_{i}$ a shortcut notation for $\operatorname{pad}[r]\left(\left|\sigma_{i}\right|\right)$.

The output of a duplexing call is thus the output of a sponge function with an input $\sigma_{0}\left\|\operatorname{pad}_{0}\right\| \sigma_{1}\left\|\operatorname{pad}_{1}\right\| \ldots \| \sigma_{i}$ and from this input the exact sequence $\sigma_{0}, \sigma_{1}, \ldots, \sigma_{i}$ can be recovered as shown in Lemma 4 below. As such, the duplex construction is as secure as the sponge construction with the same parameters. In particular, it inherits its resistance against (single-stage) generic attacks. The reference point in this case is a random oracle whose input is the sequence of inputs to the duplexing calls since the initialization.

Lemma 4. Let pad and $r$ be fixed. Then, the mapping from a sequence of binary strings $\left(\sigma_{0}, \sigma_{1}, \ldots, \sigma_{n}\right)$ with $\left|\sigma_{i}\right| \leq \rho_{\max }(\mathrm{pad}, r) \forall i$ to the binary string $s=$ $\sigma_{0}\left\|\operatorname{pad}_{0}\right\| \sigma_{1}\left\|\operatorname{pad}_{1}\right\| \ldots\left\|\operatorname{pad}_{n-1}\right\| \sigma_{n}$ is injective.

In the following sections we will show that the duplex construction is a powerful tool for building modes of use. 


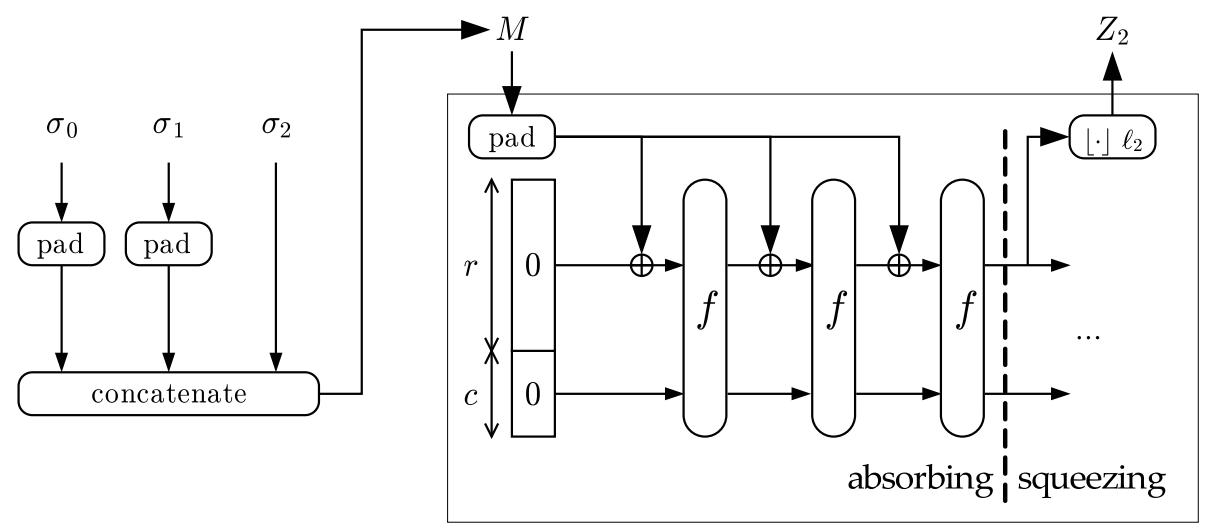

sponge

Fig. 2. Generating the output of a duplexing call with a sponge

\section{The Authenticated Encryption Mode SpongeWrap}

We propose an authenticated encryption mode SPONGEWRAP that realizes the authenticated encryption process defined in Section 2. Similarly to the duplex construction, we call an instance of the authenticated encryption mode a SpongeWrap object.

Upon initialization of a SPONGEWrap object, it loads the key $K$. From then on one can send requests to it for wrapping and/or unwrapping data. The key stream blocks used for encryption and the tags depend on the key $K$ and the data sent in all previous requests. The authenticated encryption of a sequence of header-body pairs, as described in Section 2.1 can be performed with a sequence of wrap or unwrap requests to a SPONGEWRAP object.

\subsection{Definition}

A SpongeWrap object $W$ internally uses a duplex object $D$ with parameters $f$, pad and $r$. Upon initialization of a SPONGEWRAP object, it initializes $D$ and forwards the (padded) key blocks $K$ to $D$ using mute $D$.duplexing() calls.

When receiving a $W \cdot \operatorname{wrap}(A, B, \ell)$ request, it forwards the blocks of the (padded) header $A$ and the (padded) body $B$ to $D$. It generates the cryptogram $C$ block by block $C_{i}=B_{i} \oplus Z_{i}$ with $Z_{i}$ the response of $D$ to the previous $D$.duplexing () call. The $\ell$-bit tag $T$ is the response of $D$ to the last body block (possibly extended with the response to additional blank $D$.duplexing() calls in case $\ell>\rho$ ). Finally it returns the cryptogram $C$ and the $\operatorname{tag} T$.

When receiving a $W \cdot \operatorname{unwrap}(A, C, T)$ request, it forwards the blocks of the (padded) header $A$ to $D$. It decrypts the data body $B$ block by block $B_{i}=C_{i} \oplus Z_{i}$ with $Z_{i}$ the response of $D$ to the previous $D$.duplexing() call. The response of $D$ 
to the last body block (possibly extended) is compared with the tag $T$ received as input. If the tag is valid, it returns the data body $B$; otherwise, it returns an error. Note that in implementations one may impose additional constraints, such as SPONGEWRAP objects dedicated to either wrapping or unwrapping. Additionally, the SPONGEWRAP object should impose a minimum length $t$ for the tag received before unwrapping and could break the entire session as soon as an incorrect tag is received.

Before being forwarded to $D$, every key, header, data or cryptogram block is extended with a so-called frame bit. The rate $\rho$ of the SpongeWRAP mode determines the size of the blocks and hence the maximum number of bits processed per call to $f$. Its upper bound is $\rho_{\max }(\operatorname{pad}, r)-1$ due to the inclusion of one frame bit per block. A formal definition of SPONGEWRAP is given in Algorithm 3 .

\subsection{Security}

In this section, we show the security of SPONGEWRAP against generic attacks. To do so, we proceed in two steps. First, we define a variant of ROwRAP for which the key stream depends not only on $A$ but also on previous blocks of $B$. Then, we quantify the increase in the adversary advantage when trading the random oracles $\mathcal{R} \mathcal{O}_{\mathrm{C}}$ and $\mathcal{R} \mathcal{O}_{\mathrm{T}}$ with a random sponge function and appropriate input mappings.

For a fixed block length $\rho$, let

$$
\operatorname{pre}_{i}(\overline{A, B})=\left(A^{(1)}, B^{(1)}, A^{(2)}, \ldots, B^{(n-1)}, A^{(n)},\left\lfloor B^{(n)}\right\rfloor_{i \rho}\right),
$$

i.e., the last body $B^{(n)}$ is truncated to its first $i$ blocks of $\rho$ bits. We define ROWRAP $[\rho]$ identically to ROWRAP, except that in the wrapping algorithm, we have

$$
\begin{aligned}
C^{(n)}= & \left\lfloor\mathcal{R} \mathcal{O}_{\mathrm{C}}\left(K, \operatorname{pre}_{0}(\overline{A, B})\right)\right\rfloor_{\left|B_{0}^{(n)}\right|} \oplus B_{0}^{(n)} \\
\| & \left.\mathcal{R} \mathcal{O}_{\mathrm{C}}\left(K, \operatorname{pre}_{1}(\overline{A, B})\right)\right\rfloor_{\left|B_{1}^{(n)}\right|} \oplus B_{1}^{(n)} \\
& \cdots \\
\| & \left.\mathcal{R} \mathcal{O}_{\mathrm{C}}\left(K, \operatorname{pre}_{w}(\overline{A, B})\right)\right\rfloor_{\left|B_{w}^{(n)}\right|} \oplus B_{w}^{(n)}
\end{aligned}
$$

for $B^{(n)}=B_{0}^{(n)}|| B_{1}^{(n)}|| \ldots|| B_{w}^{(n)}$ with $\left|B_{i}^{(n)}\right|=\rho$ for $i<w,\left|B_{w}^{(n)}\right| \leq \rho$ and $\left|B_{w}^{(n)}\right|>0$ if $w>0$. The unwrap algorithm $U$ is defined accordingly.

The scheme ROwRAP $[\rho]$ is as secure as ROwRAP, as expressed in the following two lemmas. We omit the proofs, as they are very similar to those of Lemma 1 and 2 .

Lemma 5. Let $\mathcal{A}\left[\mathcal{R} \mathcal{O}_{\mathrm{C}}, \mathcal{R} \mathcal{O}_{\mathrm{T}}\right]$ be an adversary having access to $\mathcal{R} \mathcal{O}_{\mathrm{C}}$ and $\mathcal{R} \mathcal{O}_{\mathrm{T}}$ and respecting the nonce requirement. Then, $\operatorname{Adv}_{\mathrm{ROwRAP}[\rho]}^{\text {priv }}(\mathcal{A}) \leq q 2^{-k}$ if the adversary makes no more than q queries to $\mathcal{R O}_{\mathrm{C}}$ or $\mathcal{R} \mathcal{O}_{\mathrm{T}}$. 


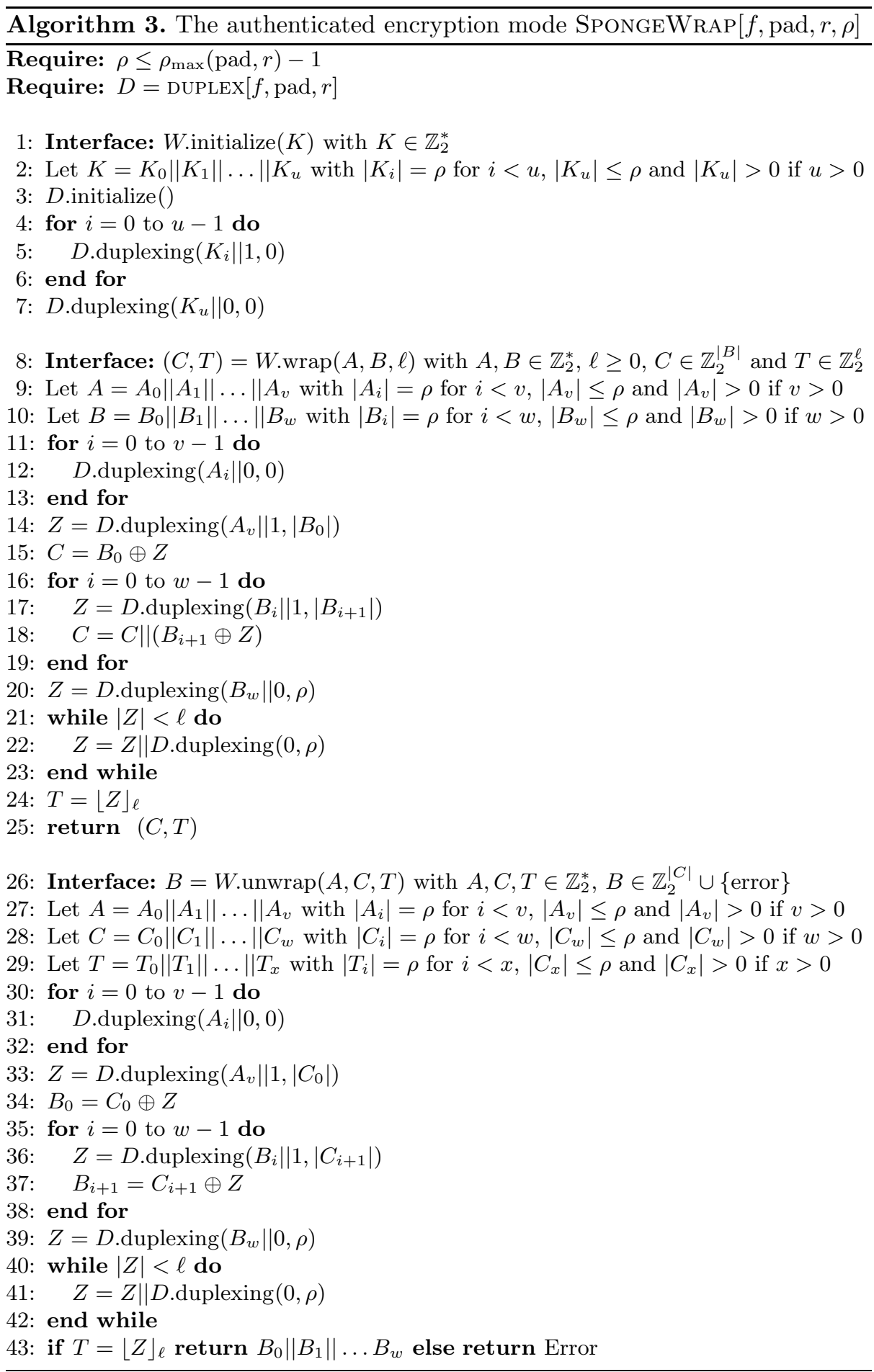


Lemma 6. Let $\mathcal{A}\left[\mathcal{R \mathcal { O } _ { \mathrm { C } }}, \mathcal{R \mathcal { O } _ { \mathrm { T } }}\right]$ be an adversary having access to $\mathcal{R O}_{\mathrm{C}}$ and $\mathcal{R} \mathcal{O}_{\mathrm{T}}$. Then, ROWRAP satisfies $\operatorname{Adv}_{\mathrm{ROWRAP}[\rho]}^{\text {auth }}(\mathcal{A}) \leq q 2^{-k}+2^{-t}$ if the adversary makes no more than q queries to $\mathcal{R} \mathcal{O}_{\mathrm{C}}$ or $\mathcal{R O}_{\mathrm{T}}$.

Clearly, ROWRAP and ROWRAP $[\rho]$ are equally secure if we implement $\mathcal{R} \mathcal{O}_{\mathrm{C}}$ and $\mathcal{R} \mathcal{O}_{\mathrm{T}}$ using a single random oracle with domain separation: $\mathcal{R} \mathcal{O}_{\mathrm{C}}(x)=\mathcal{R O}(x \| 1)$ and $\mathcal{R O}_{\mathrm{T}}(x)=\mathcal{R O}(x \| 0)$. Notice that SPONGEWRAP uses the same domain separation technique: the last bit of the input of the last duplexing call is always a 1 (resp. 0) to produce key stream bits (resp. to produce the tag). With this change, SPONGEWRAP now works like ROWRAP $[\rho]$, except that the input is formatted differently and that a sponge function replaces $\mathcal{R O}$. The next lemma focuses on the former aspect.

Lemma 7. Let $(K, \overline{A, B})$ be a sequence of strings composed by a key followed by header-body pairs. Then, the mapping from $(K, \overline{A, B})$ to the corresponding sequence of inputs $\left(\sigma_{0}, \sigma_{1}, \ldots, \sigma_{n}\right)$ to the duplexing calls in Algorithm 3 is injective.

We now have all the ingredients to prove the following theorem.

Theorem 1. The authenticated encryption mode SpOngEWRAP $[f, \operatorname{pad}, r, \rho]$ defined in Algorithm 3 satisfies

$$
\begin{aligned}
& \operatorname{Adv}_{\text {SpongeWrap }[f, \operatorname{pad}, r, \rho]}^{\text {priv }}(\mathcal{A})<q 2^{-k}+\frac{N(N+1)}{2^{c+1}} \text { and } \\
& \operatorname{Adv}_{\text {SpongeWrap }[f, \operatorname{pad}, r, \rho]}^{\text {auth }}(\mathcal{A})<q 2^{-k}+2^{-t}+\frac{N(N+1)}{2^{c+1}},
\end{aligned}
$$

against any single adversary $\mathcal{A}$ if $K \stackrel{\$}{\leftarrow} \mathbb{Z}_{2}^{k}$, tags of $\ell \geq t$ bits are used, $f$ is a randomly chosen permutation, $q$ is the number of queries and $N$ is the number of times $f$ is called.

Note that all the outputs of SPONGEWRAP are equivalent to calls to a sponge function with the secret key blocks as a prefix. So the results of [9] can also be applied to SpOngEWrap as explained in Section 3.2 .

\subsection{Advantages and Limitations}

The authenticated encryption mode SPONGEWRAP has the following unique combination of advantages:

- While most other authenticated encryption modes are described in terms of a block cipher, SPONGEWRAP only requires on a fixed-length permutation.

- It supports the alternation of strings that require authenticated encryption and strings that only require authentication.

- It can provide intermediate tags after each $W \cdot \operatorname{wrap}(A, B, \ell)$ request.

- It has a strong security bound against generic attacks with a simple proof.

- It is single-pass and requires only a single call to $f$ per $\rho$-bit block. 
- It is flexible as the bitrate can be freely chosen as long as the capacity is larger than some lower bound.

- The encryption is not expanding.

As compared to some block cipher based authenticated encryption modes, it has some limitations. First, the mode as such is serial and cannot be parallelized at algorithmic level. Some block cipher based modes do actually allow parallelization, for instance, the offset codebook (OCB) mode [27]. Yet, SpongeWrap variants could be defined to support parallel streams in a fashion similar to tree hashing, but with some overhead.

Second, if a system does not impose the nonce requirement on $A$, an attacker may send two requests $(A, B)$ and $\left(A, B^{\prime}\right)$ with $B \neq B^{\prime}$. In this case, the first differing blocks of $B$ and $B^{\prime}$, say $B_{i}$ and $B_{i}^{\prime}$, will be enciphered with the same key stream, making their bitwise XOR available to the attacker. Some block cipher based modes are misuse resistant, i.e., they are designed in such a way that in case the nonce requirement is not fulfilled, the only information an attacker can find out is whether $B$ and $B^{\prime}$ are equal or not [29]. Yet, many applications already provide a nonce, such as a packet number or a key ID, and can put it in $A$.

\subsection{An Application: Key Wrapping}

Key wrapping is the process of ensuring the secrecy and integrity of cryptographic keys in transport or storage, e.g., 2314. A payload key is wrapped with a key-encrypting key (KEK). We can use the SPONGEWRAP mode with $K$ equal to the KEK and let the data body be the payload key value. In a sound key management system every key has a unique identifier. It is sufficient to include the identifier of the payload key in the header $A$ and two different payload keys will never be enciphered with the same key stream. When wrapping a private key, the corresponding public key or a digest computed from it can serve as identifier.

\section{Other Applications of the Duplex Construction}

Authenticated encryption is just one application of the duplex construction. In this section we illustrate it by providing two more examples: a pseudo-random bit sequence generator and a sponge-like construction that overwrites part of the state with the input block rather than to XOR it in.

\subsection{A Reseedable Pseudo-random Bit Sequence Generator}

In various cryptographic applications and protocols, random bits are used to generate keys or unpredictable challenges. While randomness can be extracted from a physical source, it is often necessary to provide many more bits than the entropy of the physical source. A pseudo-random bit sequence generator (PRG) is initialized with a seed, generated in a secret or truly random way, and it 
then expands the seed into a sequence of bits. For cryptographic purposes, it is required that the generated bits cannot be predicted, even if subsets of the sequence are revealed. In this context, a PRG is similar to a stream cipher. A PRG is also similar to a cryptographic hash function when gathering entropy coming from different sources. Finally, some applications require a pseudo-random bit sequence generator to support forward security: The compromise of the current state does not enable the attacker to determine the previously generated pseudo-random bits 413.

Conveniently, a pseudo-random bit sequence generator can be reseedable, i.e., one can bring an additional source of entropy after pseudo-random bits have been generated. Instead of throwing away the current state of the PRG, reseeding combines the current state of the generator with the new seed material. In [7] a reseedable PRG was defined based on the sponge construction that implements the required functionality. The ideas behind that PRG are very similar to the duplex construction. We however show that such a PRG can be defined on top of the duplex construction.

A duplex object can readily be used as a reseedable PRG. Seed material can be fed via the $\sigma$ inputs in D.duplexing() call and the responses can be used as pseudo-random bits. If pseudo-random bits are required and there is no seed available, one can simply send blank D.duplexing() calls. The only limitation of this is that the user must split his seed material in strings of at most $\rho_{\max }$ bits and that at most $r$ bits can be requested in a single call. This limitation is removed in a more elaborate generator called SPONGEPRG presented in [8]. This mode is similar to the one proposed in [7] in that it minimizes the number of calls to $f$, although explicitly based on the duplex construction.

\subsection{The Mode Overwrite}

In [17] sponge-like constructions were proposed and cryptanalyzed. In some of these constructions, absorbing is done by overwriting part of the state by the message block rather than XORing it in, e.g., as in the hash function Grindahl [19]. These overwrite functions have the advantage over sponge functions that between calls to $f$, only $c$ bits must be kept instead of $b$. This may not be useful when hashing in a continuous fashion, as $b$ bits must be processed by $f$ anyway. However, when hashing a partial message, then putting it aside to continue later on, storing only $c$ bits may be useful on some platforms.

Defined in [8], the mode OverwRITE differs from the sponge construction in that it overwrites part of the state with an input block instead of XORing it in.

Such a mode can be analyzed by building it on top of the duplex construction. If the first $\rho$ bits of the state are known to be $Z$, overwriting them with a message block $P_{i}$ is equivalent to XORing in $Z \oplus P_{i}$. In [8], we have proven that the security of OvERWRITE is equivalent to that of the sponge construction with the same parameter, but at a cost of 2 bits of bitrate (or equivalently, of capacity): one for the padding rule (assuming pad10* is used) and one for a frame bit. 


\section{$7 \quad$ A Flexible and Compact Padding Rule}

Sponge functions and duplex objects feature the nice property of allowing a range of security-performance trade-offs, via capacity-rate pairs, using the same fixed permutation $f$. To be able to fully exploit this property in the scope of the duplex construction, and for performance reasons, the padding rule should be compact and should be suitable for a family of sponge functions with different rates.

For a given capacity and width, the padding reduces the maximum bitrate of the duplex construction, as in Eq. (4). To minimize this effect, especially when the width of the permutation is relatively small, one should look for the most compact padding rule. The sponge-compliant padding scheme (see Section 3) with the smallest overhead is the well-known simple reversible padding, which appends a single 1 and the smallest number of zeroes such that the length of the result is a multiple of the required block length. We denote it by pad $10^{*}[r](M)$. It satisfies $\rho_{\max }\left(\operatorname{pad} 10^{*}, r\right)=r-1$ and hence has only one bit of overhead.

When considering the security of a set of sponge functions that make use of the same permutation $f$ but with different bitrates, simple reversible padding is not sufficient. The indifferentiability proof of [6] actually only covers the indifferentiability of a single sponge function instance from a random oracle. As a solution, we propose the multi-rate padding, denoted pad10*1[r] $(|M|)$, which returns a bitstring $10^{q} 1$ with $q=(-|M|-2) \bmod r$. This padding is spongecompliant and has $\rho_{\max }\left(\operatorname{pad} 10^{*} 1, r\right)=r-2$. Hence, this padding scheme is compact as the duplex-level maximum rate differs from the sponge-level rate by only two bits. Furthermore, in Theorem 2 we will show it is sufficient for the indifferentiability of a set of sponge functions. The intuitive idea behind this is that, with the pad10*1 padding scheme, the last block absorbed has a bit with value 1 at position $r-1$, while any other function of the family with $r^{\prime}<r$ this bit has value 0 .

Besides having a compact padding rule, it is also useful to allow the sponge function to have specific bitrate values. In many applications one prefers to have block lengths that are a multiple of 8 or even higher powers of two to avoid bit shifting or misalignment issues. With modes using the duplex construction, one has to distinguish between the mode-level block size and the bitrate of the underlying sponge function. For instance in the authenticated encryption mode SpongeWrap, the block size is at most $\rho_{\max }(\operatorname{pad}, r)-1$. To have a block size with the desired value, it suffices to take a slightly higher value as bitrate $r$; hence, the sponge-level bitrate may no longer be a multiple of 8 or of a higher power of two. Therefore it is meaningful to consider the security of a set of sponge functions with common $f$ and different bitrates, including bitrates that are not multiples of 8 or of a higher power of two. For instance, the mode SpongeWraP could be based on $\operatorname{KeCCAK}[r=1027, c=573]$ so as to process application-level blocks of $\rho_{\max }\left(\operatorname{pad} 10^{*} 1,1027\right)-1=1024$ bits $[10$.

Regarding the indifferentiability of a set of sponge functions, it is clear that the best one can achieve is bounded by the strength of the sponge construction with the lowest capacity (or, equivalently, the highest bitrate), as an adversary can 
always just try to differentiate the weakest construction from a random oracle. The next theorem states that we achieve this bound by using the multi-rate padding.

Theorem 2. Given a random permutation (or transformation) $f$, differentiating the array of sponge functions SPONGE $[f$, pad10*1, $r]$ with $0<r \leq r_{\max }$ from an array of independent random oracles $\left(\mathcal{R} \mathcal{O}_{r}\right)$ has the same advantage as differentiating SPONGE $\left[f\right.$, pad $\left.10^{*}, r_{\max }\right]$ from a random oracle.

\section{References}

1. Aumasson, J.-P., Henzen, L., Meier, W., Naya-Plasencia, M.: Quark: A lightweight hash. In: Mangard and Standaert [20], pp. 1-15

2. Bellare, M., Namprempre, C.: Authenticated Encryption: Relations among Notions and Analysis of the Generic Composition Paradigm. In: Okamoto, T. (ed.) ASIACRYPT 2000. LNCS, vol. 1976, pp. 531-545. Springer, Heidelberg (2000)

3. Bellare, M., Rogaway, P.: Random oracles are practical: A paradigm for designing efficient protocols. In: ACM (ed.) ACM Conference on Computer and Communications Security 1993, pp. 62-73 (1993)

4. Bellare, M., Yee, B.: Forward-security in private-key cryptography. Cryptology ePrint Archive, Report 2001/035 (2001), http://eprint.iacr.org/

5. Bertoni, G., Daemen, J., Peeters, M., Van Assche, G.: Sponge functions. In: Ecrypt Hash Workshop (May 2007), public comment to NIST, from http://www.csrc.nist.gov/pki/HashWorkshop/ Public_Comments/2007_May.html

6. Bertoni, G., Daemen, J., Peeters, M., Van Assche, G.: On the Indifferentiability of the Sponge Construction. In: Smart, N.P. (ed.) EUROCRYPT 2008. LNCS, vol. 4965, pp. 181-197. Springer, Heidelberg (2008),

http://sponge.noekeon.org/

7. Bertoni, G., Daemen, J., Peeters, M., Van Assche, G.: Sponge-based pseudorandom number generators. In: Mangard and Standaert [20], pp. 33-47

8. Bertoni, G., Daemen, J., Peeters, M., Van Assche, G.: Duplexing the sponge: singlepass authenticated encryption and other applications. Cryptology ePrint Archive, Report 2011/499 (2011), http://eprint.iacr.org/

9. Bertoni, G., Daemen, J., Peeters, M., Van Assche, G.: On the security of the keyed sponge construction. In: Symmetric Key Encryption Workshop (SKEW) (February 2011)

10. Bertoni, G., Daemen, J., Peeters, M., Van Assche, G.: The KECCAK reference (January 2011), http://keccak.noekeon.org/

11. Biryukov, A. (ed.): FSE 2007. LNCS, vol. 4593. Springer, Heidelberg (2007)

12. Bogdanov, A., Knežević, M., Leander, G., Toz, D., Varıcı, K., Verbauwhede, I.: SPOngent: A Lightweight Hash Function. In: Preneel, B., Takagi, T. (eds.) CHES 2011. LNCS, vol. 6917, pp. 312-325. Springer, Heidelberg (2011)

13. Desai, A., Hevia, A., Yin, Y.L.: A Practice-Oriented Treatment of Pseudorandom Number Generators. In: Knudsen, L.R. (ed.) EUROCRYPT 2002. LNCS, vol. 2332, pp. 368-383. Springer, Heidelberg (2002)

14. Dworkin, M.: Request for review of key wrap algorithms. Cryptology ePrint Archive, Report 2004/340 (2004), http://eprint.iacr.org/ 
15. ECRYPT Network of excellence, The SHA-3 Zoo (2011), http://ehash.iaik.tugraz.at/index.php/The_SHA-3_Zoo

16. Ferguson, N., Whiting, D., Schneier, B., Kelsey, J., Lucks, S., Kohno, T.: Helix: Fast Encryption and Authentication in a Single Cryptographic Primitive. In: Johansson, T. (ed.) FSE 2003. LNCS, vol. 2887, pp. 330-346. Springer, Heidelberg (2003)

17. Gorski, M., Lucks, S., Peyrin, T.: Slide Attacks on a Class of Hash Functions. In: Pieprzyk, J. (ed.) ASIACRYPT 2008. LNCS, vol. 5350, pp. 143-160. Springer, Heidelberg (2008)

18. Guo, J., Peyrin, T., Poschmann, A.: The PHOTON Family of Lightweight Hash Functions. In: Rogaway, P. (ed.) CRYPTO 2011. LNCS, vol. 6841, pp. 222-239. Springer, Heidelberg (2011)

19. Knudsen, L., Rechberger, C., Thomsen, S.: The Grindahl hash functions. In: Biryukov [11], pp. 39-57

20. Mangard, S., Standaert, F.-X. (eds.): CHES 2010. LNCS, vol. 6225. Springer, Heidelberg (2010)

21. Maurer, U., Renner, R., Holenstein, C.: Indifferentiability, Impossibility Results on Reductions, and Applications to the Random Oracle Methodology. In: Naor, M. (ed.) TCC 2004. LNCS, vol. 2951, pp. 21-39. Springer, Heidelberg (2004)

22. Muller, F.: Differential attacks against the Helix stream cipher. In: Roy and Meier [30], pp. 94-108

23. NIST, AES key wrap specification (November 2001)

24. Paul, S., Preneel, B.: Solving Systems of Differential Equations of Addition. In: Boyd, C., González Nieto, J.M. (eds.) ACISP 2005. LNCS, vol. 3574, pp. 75-88. Springer, Heidelberg (2005)

25. Ristenpart, T., Shacham, H., Shrimpton, T.: Careful with Composition: Limitations of the Indifferentiability Framework. In: Paterson, K.G. (ed.) EUROCRYPT 2011. LNCS, vol. 6632, pp. 487-506. Springer, Heidelberg (2011)

26. Rogaway, P.: Authenticated-encryption with associated-data. In: ACM Conference on Computer and Communications Security 2002 (CCS 2002), pp. 98-107. ACM Press (2002)

27. Rogaway, P., Bellare, M., Black, J.: OCB: A block-cipher mode of operation for efficient authenticated encryption. ACM Trans. Inf. Syst. Secur. 6(3), 365-403 (2003)

28. Rogaway, P., Bellare, M., Black, J., Krovetz, T.: OCB: A block-cipher mode of operation for efficient authenticated encryption. In: CCS 2001: Proceedings of the 8th ACM Conference on Computer and Communications Security, pp. 196-205. ACM, New York (2001)

29. Rogaway, P., Shrimpton, T.: A Provable-Security Treatment of the KeyWrap Problem. In: Vaudenay, S. (ed.) EUROCRYPT 2006. LNCS, vol. 4004, pp. 373-390. Springer, Heidelberg (2006)

30. Roy, B., Meier, W. (eds.): FSE 2004. LNCS, vol. 3017. Springer, Heidelberg (2004)

31. Whiting, D., Schneier, B., Lucks, S., Muller, F.: Fast encryption and authentication in a single cryptographic primitive, ECRYPT Stream Cipher Project Report 2005/027 (2005), http://www.ecrypt.eu.org/stream/phelixp2.html

32. Wu, H., Preneel, B.: Differential-linear attacks against the stream cipher Phelix. In: Biryukov [11], pp. 87-100

33. Ågren, M., Hell, M., Johansson, T., Meier, W.: A new version of Grain128 with authentication. In: Symmetric Key Encryption Workshop, SKEW (February 2011) 